\title{
Optical imaging of the breast: evaluation of deoxyhemoglobin concentration alteration in 166 patients with suspicious breast lesions
}

Antonella Petrillo ${ }^{1 *}$, Orlando Catalano ${ }^{1}$, Roberta Fusco ${ }^{1}$, Salvatore Filice ${ }^{1}$, Paolo Vallone ${ }^{1}$, Sergio Setola ${ }^{1}$, Vincenza Granata', Concetta Raiano', Franca Avino², Maurizio Di Bonito ${ }^{3}$ and Gerardo Botti ${ }^{3}$

\begin{abstract}
Background: We investigated the performance of optical imaging evaluating deoxyhemoglobin concentration alteration (DeHCA) in breast tissues.

Methods: We enrolled all consecutive patients from January 2015 to October 2016 with clinically suspicious and/or BI-RADS grade 3-5 lesions at mammography or ultrasound (US). Patients underwent optical imaging (ComfortScan) to evaluate for DeHCA. The reference standard was pathology from a surgical specimen for malignant lesions, pathology from a surgical specimen or core needle biopsy for benign lesions, and negative follow-up for contralateral negative breasts. Non-parametric statistics, receiver operating characteristic, and linear discrimination analyses were performed.

Results: Of 334 enrolled patients, 168 (50\%) were excluded for technical problems and 166 (50\%) (median age 52 years) were analyzed totaling 331 breasts and 176 lesions. Of these, 75 were benign (median size $19 \mathrm{~mm}$ ) and 101 malignant (median size $20 \mathrm{~mm}$ ). The median DeHCA score in malignant lesions $(0.95$, interquartile range [IQR] $1.00-0.87)$ was higher $(p<0.001)$ than in benign lesions $(0.80$, IQR 0.95-0.70). Using the optimal cutoff $(0.85)$, DeHCA score was less accurate than mammography, US, and their combination, with 78\% sensitivity, 52\% specificity, 40\% positive predictive value (PPV), and 85\% negative predictive value (NPV); using a 0.8 cutoff, sensitivity reached 93\% and NPV 91\%, but specificity fell to $32 \%$ and PPV to $37 \%$. The accuracy of DeHCA score linearly combined with mammography or US was higher than that of DeHCA score alone $(p<0.001)$ and not significantly higher than that of mammography or US alone.
\end{abstract}

Conclusions: DeHCA score was significantly higher in malignant than in benign lesions, but its accuracy was significantly lower than that of mammography or US. Future refinements are needed.

Keywords: Breast cancer, Breast ultrasound, Deoxyhemoglobin concentration alteration, Mammography, Optical imaging

\footnotetext{
* Correspondence: a.petrillo@istitutotumori.na.it

${ }^{1}$ Radiology Unit, Dipartimento di Supporto ai Percorsi Oncologici Area

Diagnostica, Istituto Nazionale Tumori - IRCCS - Fondazione G. Pascale, Via

Mariano Semmola, Naples, Italy

Full list of author information is available at the end of the article
} 


\section{Key points}

- Deoxyhemoglobin concentration alteration (DeHCA) score in malignant lesions was significantly higher than that in benign lesions

- The accuracy of DeHCA score was significantly lower than that of mammography and/or ultrasound (US)

- DeHCA score accuracy linearly combined with mammography or US was significantly higher than DeHCA score alone, but not significantly higher than mammography or US alone.

\section{Background}

Breast cancer is the most common female malignancy and the second leading cause of death from cancer among women in Europe [1]. In the United States, the estimated number of new breast cancer cases and deaths in 2017 was 318.590 and 41.070, respectively [1], with an increasing number of cases affecting young women characterized by dense breasts. Early detection of breast cancer can reduce mortality and increase survival and there is a general agreement about the correlation between tumor size at diagnosis and survival; therefore, a delay in diagnosis may negatively affect prognosis [2].

Mammography is currently the gold standard screening test for breast cancer [3]. However, it has important limitations, wherein a higher breast density, especially in younger women, can represent a major obstacle for the detection of small tumors [4, 5]. Kerlikowske et al. [6] reported that age has a considerable influence on the sensitivity of mammography, which is higher (87\%) among women aged 60-69 and lower (68\%) among women aged 30-39. Breast ultrasound (US) has an additional role, particularly in young women, yet has a variable reported accuracy due to its operator-dependent nature [7]. Breast magnetic resonance imaging has become an essential tool in high-risk screening, but is not suitable for large scale screening in the average-risk population [6, 8-10]. At present, no single technology allows for the optimal screening of women aged 40-69, with the combined sequence of mammography followed by US being frequently adopted in the case of negative mammography in dense breasts. In this context, new technologies for breast cancer diagnosis deserve attention, especially those that are radiation-free, either as stand-alone or for combined use with other technologies not exposing the breast to ionizing radiation.

Transillumination of the breast dates back to the 1920s [11]. However, its low sensitivity and specificity has limited its clinical use. With progress in photonic technologies and mathematic modeling of light propagation through tissues, optical imaging has evolved to a stage that allows its evaluation in a clinical setting $[11,12]$.

Dynamic optical imaging is based on the detection and analysis of red light transmission through the breast tissue and recording of the transitory responses of the tissue due to compression inducing changes in bloodflow volume. Such pressure stimulus results in the dynamic behavior of optical properties of the tissue, creating various dynamic profiles in regions with abnormal vascularization. Preliminary results in favor of this approach in young women were published in 2005 [13]. In 2009, dynamic optical imaging was evaluated in 46 patients, wherein the number of suspicious pixels in 12 benign lesions was significantly lower than that in 35 malignant lesions, with a sensitivity, specificity, and accuracy of $74 \%, 92 \%$, and $79 \%$, respectively [14]. Cheng et al. [15] performed dynamic optical imaging in $62 \mathrm{pa-}$ tients and obtained a sensitivity and specificity of $93 \%$ and $45 \%$, respectively, in comparison with sensitivity and specificity values of $84 \%$ and $62 \%$ achieved with mammography. A recent work by D'Aiuto et al. [16] proposed a score system for the interpretation of dynamic optical images resulting in a sensitivity of $80 \%$ and a specificity of $87 \%$ in 113 patients preoperatively.

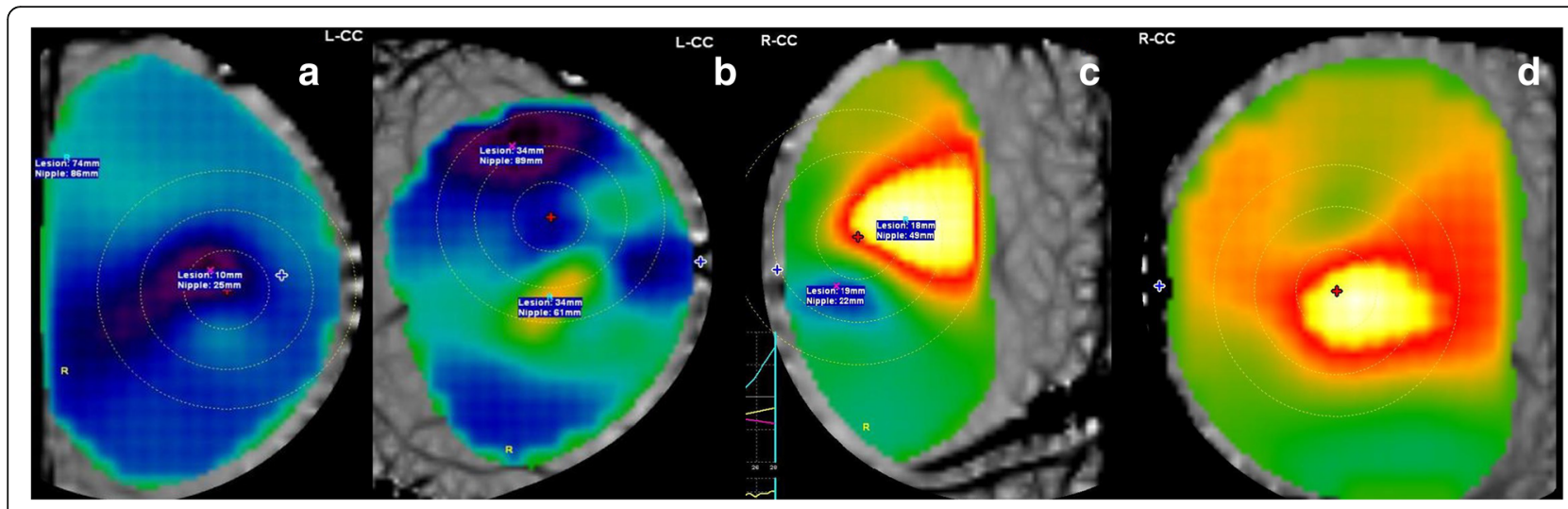

Fig. 1 Focality description: (a) blue diffusion area; (b) area with different colors (violet present); (c) area with different colors (no violet); (d) no focality 


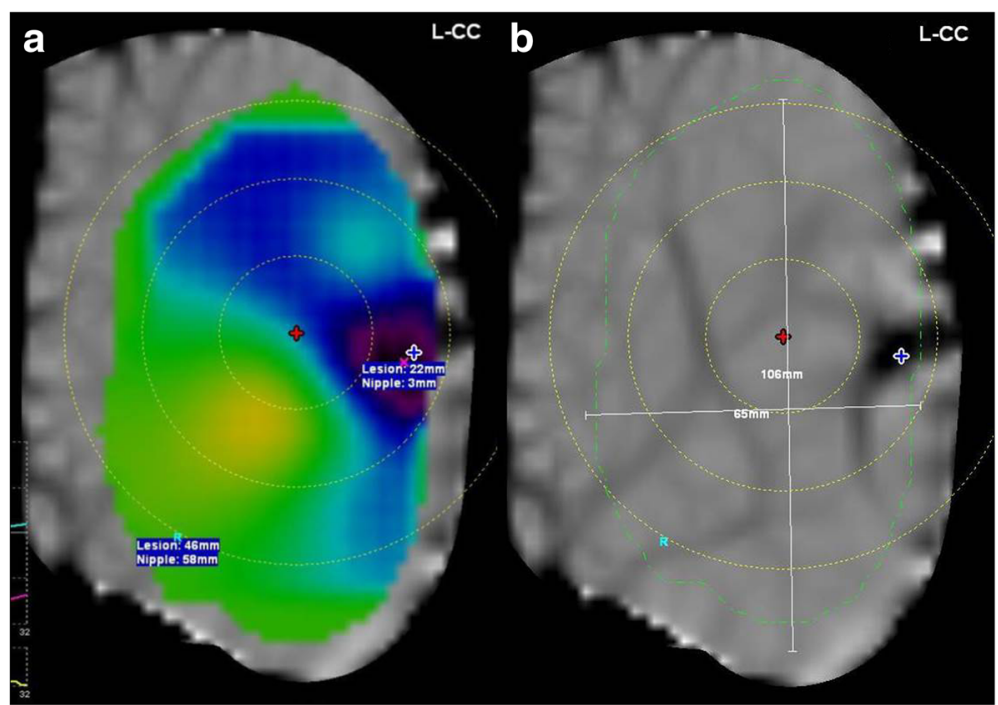

Fig. 2 Blue nipple: (a) overlap between nipple (blue cross) and lesion marker; (b) dark spot at the nipple

The aim of this study is to determine the diagnostic performance of an evolution of dynamic breast optical technology in patients with breast lesions using a score system and the combination of dynamic breast optical imaging and conventional imaging (mammography or US). The study was conducted in the context of a deoxyhemoglobin concentration alteration (DeHCA) project that assesses new applications of optical technologies in breast cancer diagnosis based on the evaluation of abnormal values of the DeHCA biomarker.

\section{Methods}

\section{Study population}

The study was performed at a National Cancer Center in Naples, Italy (Istituto Nazionale Tumori IRCCS Fondazione G. Pascale). The institutional review board approved the protocol (authorization number N. 718 on
October 9, 2014). Informed consent was obtained from all patients. The study was performed in accordance with the current version of the Declaration of Helsinki and the International Conference on Harmonization of Good Clinical Practice Guidelines.

After a training phase on DeHCA Optical Image Processing performed on more than 150 patients, we prospectively enrolled 334 consecutive patients from January 2015 to October 2016. All patients were considered eligible when they showed suspicious breast lesions at clinical examination, and/or were evaluated as Breast Imaging Reporting and Data System (BI-RADS) score 3, 4 , or 5 at mammography and/or US, were scheduled for fine needle aspiration cytology or core biopsy, and were able to be followed-up for 2 years.

Exclusion criteria were (1) the presence of a pacemaker or other devices in the chest wall; (2) an

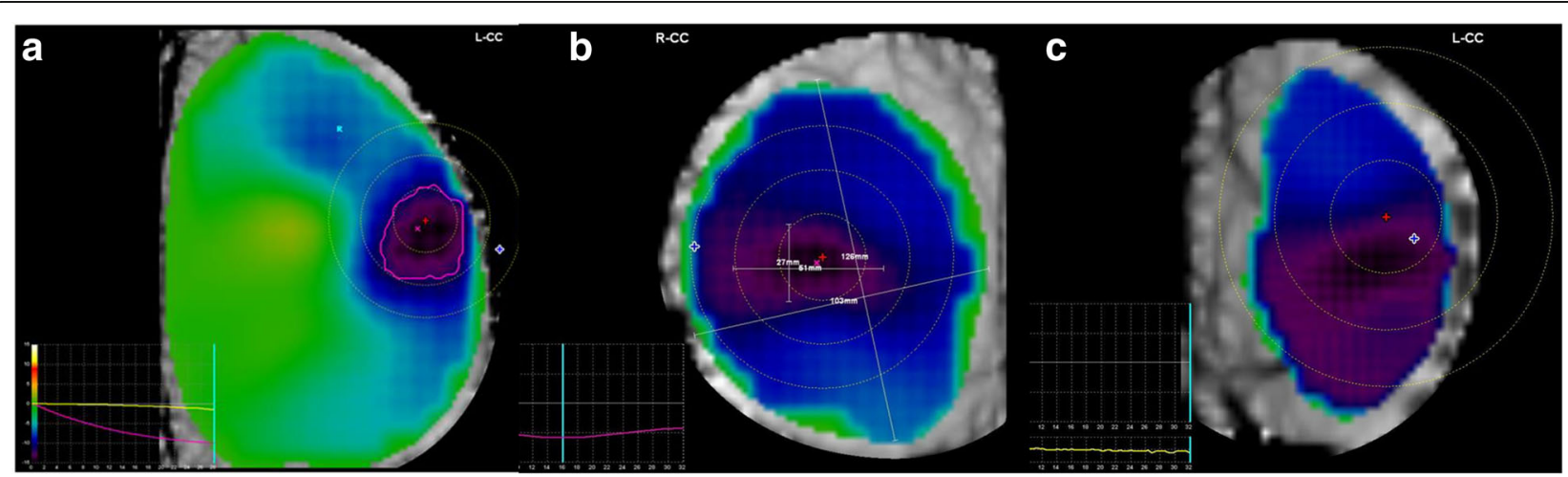

Fig. 3 Diffusion/sharpness of the focal area: (a) sharp focal area; (b) intermediate focal area; (c) diffused focal area 


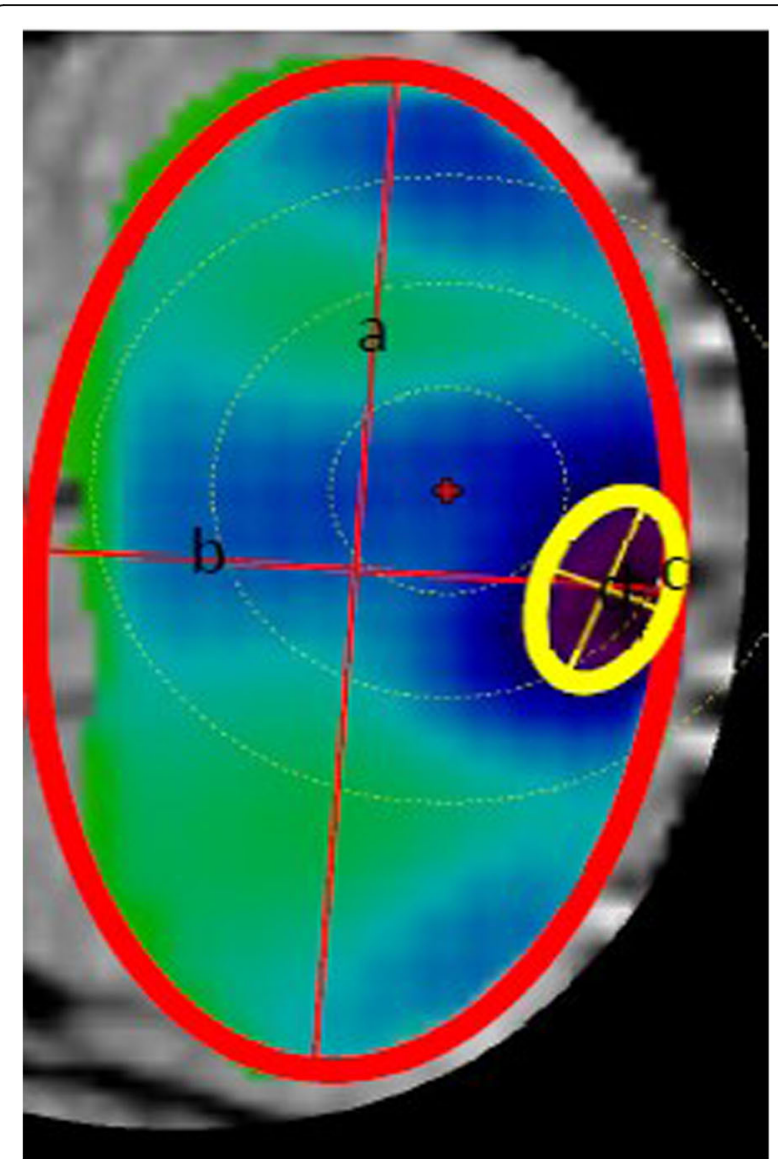

Fig. 4 Focal area dimension: calculation of both the general colored area (modeled by the red ellipse) and the lesion area (modeled by the yellow ellipse)

inability to keep upright immobility during the examination; (3) inflammatory skin diseases (psoriasis, eczema, etc.); (4) pregnancy or breast-feeding; (5) presence of tattoos on the breasts; (6) non-removable drilling at the nipple; (7) internal/external devices preventing from correct patient positioning; (8) particular physical shapes of the breast (e.g., too small breasts); and (9) a history of allergic reaction to silicone, the material from which the membrane used for breast compression in the optical imaging device is made. All patients underwent blind DeHCA optical image processing in the preoperative workflow. Contralateral negative breasts confirmed by a median follow-up time of 18 months (range 9-26 months) were included in the analysis to have a sufficiently large base for the evaluation of DeHCA specificity and positive predictive value (PPV).

\section{Mammography and US}

Mammography was performed using a screen-film Senographe DMR unit (General Electric Healthcare, Milwaukee, WI, USA). Standard bilateral cranio-caudal and medio-lateral oblique mammograms were obtained. Further dedicated mammograms (i.e., magnification, spot compression, or other additional views) were obtained when necessary.

Breast US was performed using a MyLab 70 unit (Esaote, Genova, Italy), equipped with a broadband linear array transducer $(5.5-12.5 \mathrm{MHz}$, generally employed at $10 \mathrm{MHz}$ ), which permitted a transverse resolution of $0.5 \mathrm{~mm}$ and a lateral resolution of $1 \mathrm{~mm}$ or less. Vertical, horizontal, radial, and antiradial scans of both breasts were obtained. Scanning was always extended to both axillary regions. When examining a lesion, a single focus was placed at the level of the deep aspect of the finding. The gain curve was adjusted to the depth of the lesion, while attempting to avoid artifacts. The vascular architecture of lesions identified at gray-scale B-mode imaging was investigated with color-Doppler with settings allowing for maximal sensitivity to slow flows.

Mammograms were evaluated by one of two breast radiologists with 13 and 23 years of experience, respectively. US was performed and interpreted by one of two radiologists with 15 and 23 years of experience in breast imaging, respectively. The BI-RADS categorical scoring system was used [17] to analyze images by mammography or US.
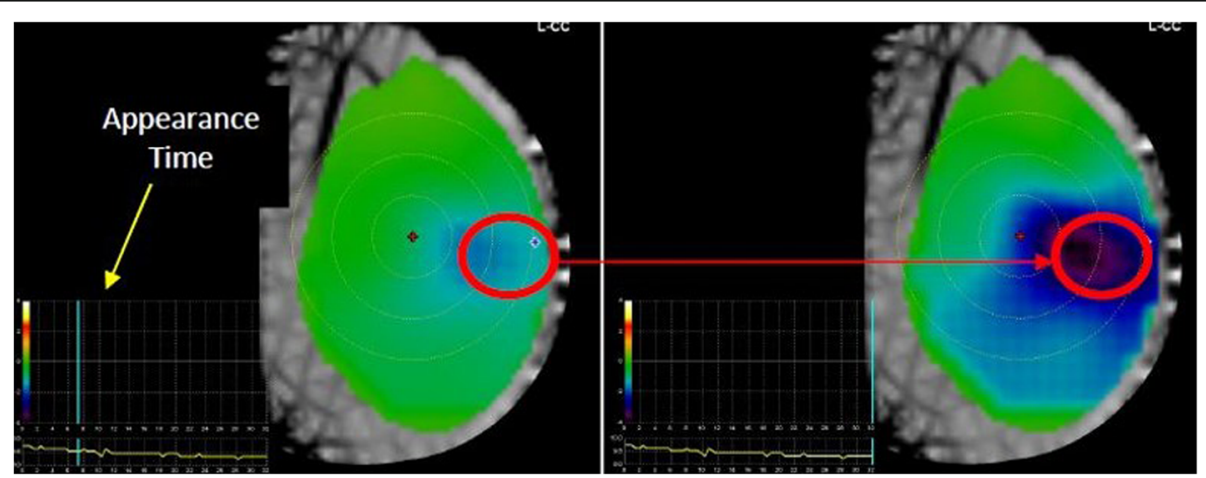

Fig. 5 Appearance time of the focal area 


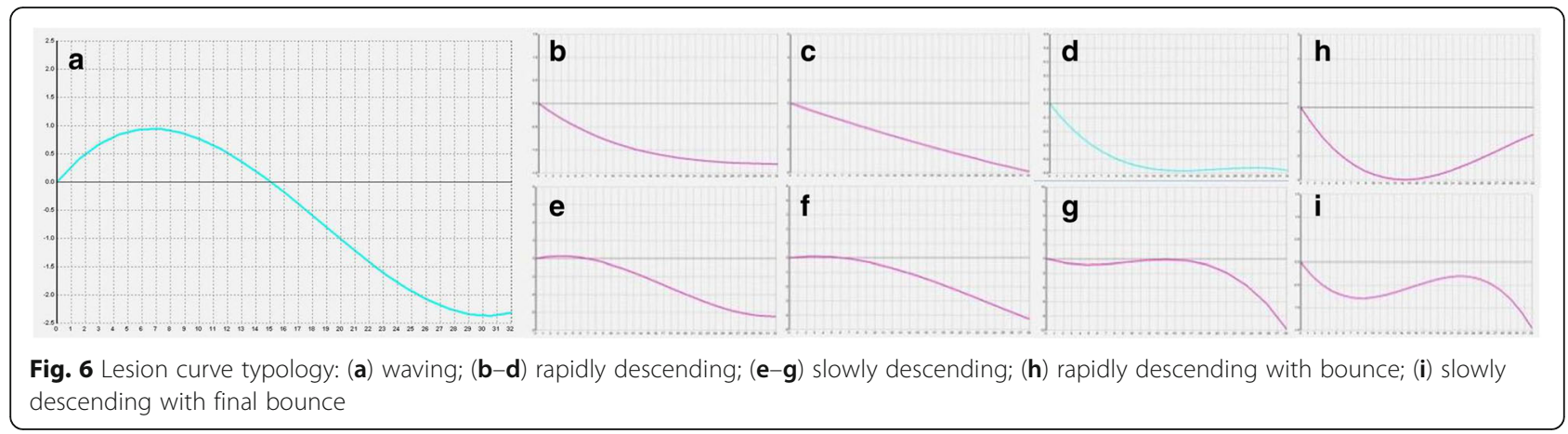

\section{DeHCA optical image processing}

The DeHCA optical image processing is an evolution of dynamic optical imaging that effectively evaluates DeHCA values over time in response to a pressure stimulus as a biomarker to be correlated with tissue neoangiogenesis. We used the ComfortScan system (DOBI Medical International, Massachusetts, USA) for optical image acquisition.

This system is an advanced optical digital imaging device that uses high-intensity, light-emitting diodes (LEDs) and gentle external pressure to highlight areas with vascular abnormalities. The high-intensity LEDs transmit red light through the breast. If the light encounters a region with neoangiogenesis, it is absorbed or scattered differently than in other regions of the breast, as a result of differences in concentrations of oxygenated and deoxygenated hemoglobin. The system is composed of three physical assemblies, namely the $\mathrm{C}$-arm assembly, the controller, and the computer system. Technical details have been previously described $[13,14]$.

The patients stand in front of the machine and the breast is positioned onto the panel of the C-arm assembly. The LEDs illuminate the breast and the light (wavelength $640 \mathrm{~nm}$ ) is transmitted through the tissues and quantified on the other side by a charge coupled device

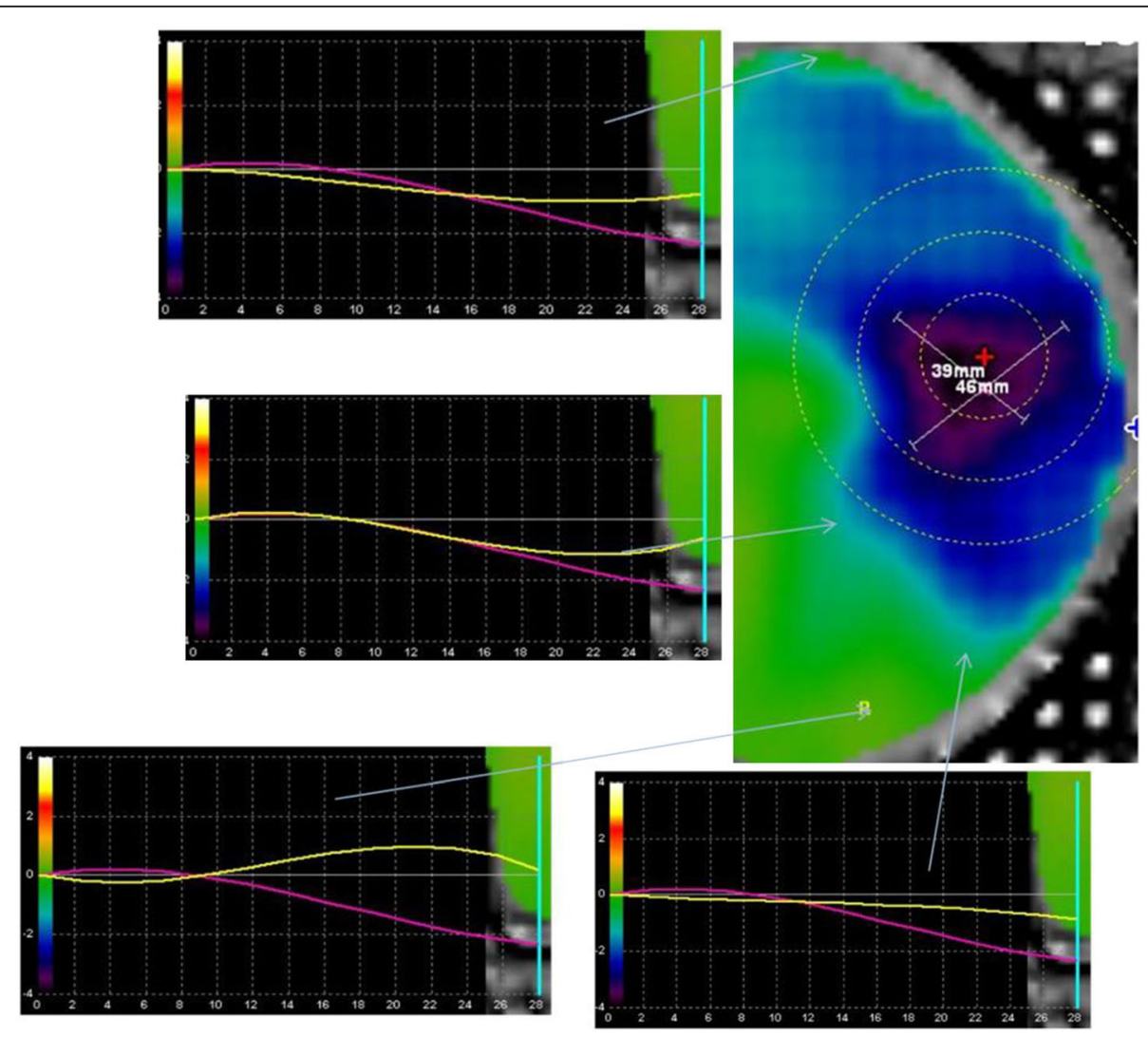

Fig. 7 Comparison between the lesion curve and curves in other regions of the breast 


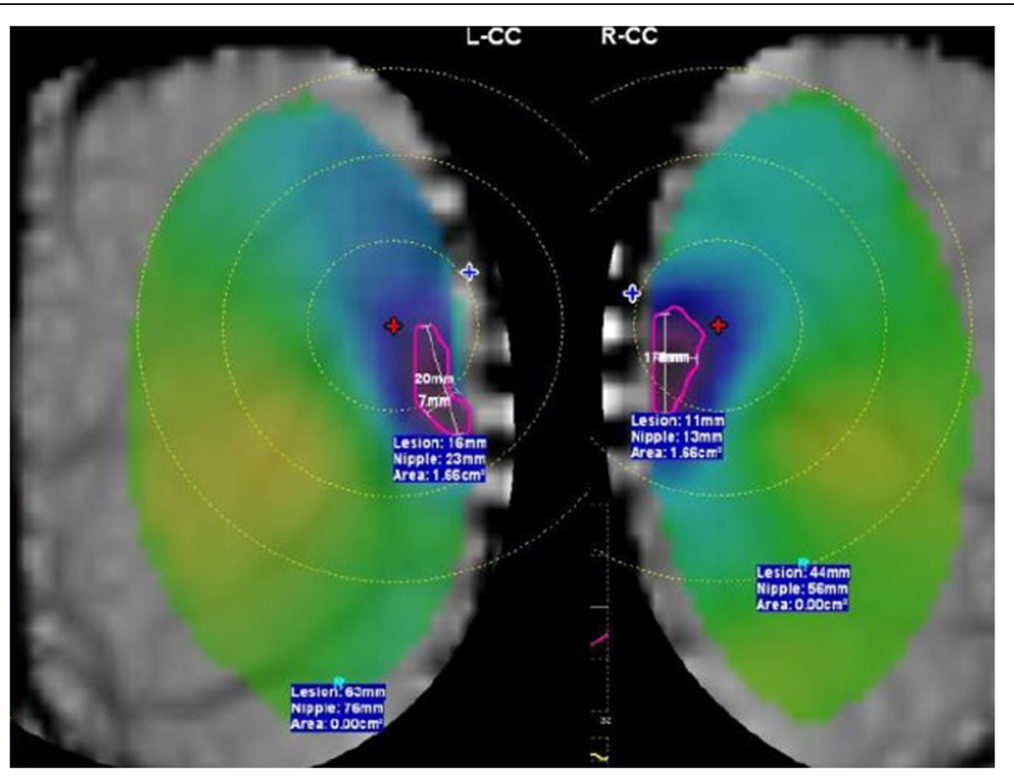

Fig. 8 Bilaterality example: the comparison between the images of the two breasts show similar behavior

Table 1 Parameters for DeHCA score calculation

\begin{tabular}{|c|c|c|c|}
\hline Parameter & State & Score & Acronym \\
\hline \multirow[t]{2}{*}{ Focality description } & Absent & 0 & FOC1 \\
\hline & Present & 1 & \\
\hline \multirow[t]{2}{*}{ Blue nipple } & Yes & 0 & BN \\
\hline & No & 1 & \\
\hline \multirow[t]{2}{*}{ Stability/mobility of focality (Epicenter) } & Mobile & 0 & EPI \\
\hline & Stable & 1 & \\
\hline \multirow[t]{3}{*}{ Diffusion/sharpness of the focal area } & Diffuse & 0 & FOC2 \\
\hline & Intermediate & 0.95 & \\
\hline & Sharp & 1 & \\
\hline \multirow[t]{3}{*}{ Focal area/total area } & Area $>20 \%$ & 0 & $A R$ \\
\hline & $10 \%<$ Area $<20 \%$ & 0.85 & \\
\hline & Area $<10 \%$ & 1 & \\
\hline \multirow[t]{2}{*}{ Bilaterality } & Yes & 0 & BIL \\
\hline & No & 1 & \\
\hline \multirow[t]{2}{*}{ Similarity } & Yes & 0.8 & SIM \\
\hline & No & 1 & \\
\hline \multirow[t]{3}{*}{ Appearance time (t) } & $t>15$ & 0.85 & TEMP \\
\hline & $10<t \leq 15$ & 0.92 & \\
\hline & $t \leq 10$ & 1 & \\
\hline \multirow[t]{3}{*}{ DeHCA score $=$ FOC1 $\times$ BN $\times \mathrm{EPI} \times \mathrm{FOC2} \times \mathrm{AR} \times \mathrm{BIL} \times \mathrm{SIM} \times \mathrm{TEMP}$} & \multicolumn{3}{|c|}{$0<$ score $<0.8$ (low risk) } \\
\hline & \multicolumn{3}{|c|}{$0.8<$ score $<0.9$ (intermediate risk) } \\
\hline & \multicolumn{3}{|c|}{$0.9<$ score $<1$ (high risk) } \\
\hline
\end{tabular}


Table 2 Histotype of breast lesions in the studied population

\begin{tabular}{llll}
\hline Disease & Subtype & $\begin{array}{l}\text { Number of } \\
\text { breasts }\end{array}$ & Percentage \\
\hline Non-malignant & Adenosis & 10 & 13.3 \\
& Dysplasia & 14 & 18.7 \\
& Fibroadenoma & 30 & 40.0 \\
& Inflammation & 9 & 12.0 \\
& Ductal hyperplasia & 9 & 12.0 \\
& Benign phyllodes & 1 & 1.3 \\
& Lobular carcinoma in situ & 2 & 2.7 \\
& Total & 75 & 100 \\
& Ductal carcinoma in situ & 3 & 3.0 \\
& Invasive ductal carcinoma & 76 & 75.2 \\
& Invasive cribriform carcinoma & 1 & 1.0 \\
& Invasive lobular carcinoma & 13 & 12.9 \\
& Invasive mucinous carcinoma & 1 & 1.0 \\
& Invasive tubular carcinoma & 5 & 5.0 \\
& Carcinomatous mastitis & 1 & 1.0 \\
& Paget disease & 1 & 1.0 \\
& Total & 101 & 100 \\
\hline
\end{tabular}

(CCD) camera. In the acquisition window of the operating software, the operator can mark the region of interest placing two pointers indicating the nipple and where the lesion is supposed to be.

A soft transparent silicone membrane is placed in contact with the upper surface of the breast and then inflated under computer control during the exam. The pressure is set to $5 \mathrm{mmHg}$ for the first $15 \mathrm{~s}$ of the scan, raised to $10 \mathrm{mmHg}$ over the next $30 \mathrm{~s}$ (dynamic sequence), and decreased back to $5 \mathrm{mmHg}$ for the final 15 s. Overall, 45 frames are acquired ( 5 baseline before applying pressure and 40 during the dynamic sequence). The transmitted light is detected by the CCD camera throughout the scan and recorded by the computer to generate the dynamic angiogenic signature, which is a sequence of cranial-caudal images. The analysis of this dynamic angiogenic signature identifies changes in local blood perfusion and oxygen saturation as variations in image contrast and identifies areas of pathologic interest that present an increase of both blood volume and depletion of blood oxygen with a reduction of the amount of light reaching the CCD camera [18]. This feature appears like an area of decreased intensity (dark blue) or highly decreased intensity (purple) of color contrast. ComfortScan acquisition time and map generation is equal to $90-120 \mathrm{~s}$.

ComfortScan images were reviewed blindly from clinical data and from mammography and US results. The

Table 3 Association between DeHCA score and prognostic factors

\begin{tabular}{|c|c|c|c|c|c|}
\hline \multirow[t]{2}{*}{ Patients' DeHCA score } & \multicolumn{2}{|l|}{$<0.8$} & \multicolumn{2}{|l|}{$\geq 0.8$} & \multirow[t]{2}{*}{$p^{a}$} \\
\hline & Number & Percentage & Number & Percentage & \\
\hline$\overline{\text { Age }}$ & & & & & $<0.001$ \\
\hline$\leq 40$ & 23 & 47.9 & 25 & 52.1 & \\
\hline $40-49$ & 34 & 33.3 & 68 & 66.7 & \\
\hline $50-59$ & 8 & 10.5 & 68 & 89.5 & \\
\hline$\geq 60$ & 10 & 9.5 & 95 & 90.5 & \\
\hline Tumor grading & & & & & 0.883 \\
\hline । & 1 & 20.0 & 4 & 80.0 & \\
\hline$\|$ & 3 & 5.2 & 55 & 94.8 & \\
\hline III & 1 & 8.3 & 11 & 91.7 & \\
\hline Herceptest & & & & & 0.470 \\
\hline Positive & 0 & 0 & 13 & 100 & \\
\hline Negative & 7 & 11.7 & 57 & 88.3 & \\
\hline Receptor status (ER/PR) & & & & & 0.031 \\
\hline Positive & 2 & 4 & 48 & 96 & \\
\hline Negative & 4 & 26.7 & 11 & 73.3 & \\
\hline Ki67 & & & & & 0.082 \\
\hline Positive & 0 & 0 & 28 & 100 & \\
\hline Negative & 7 & 14.9 & 40 & 85.1 & \\
\hline
\end{tabular}

${ }^{a} \mathrm{X}^{2}$ test with Yates' correction 
Table 4 BI-RADS diagnostic categories distribution in the studied population

\begin{tabular}{|c|c|c|c|}
\hline & $\begin{array}{l}\text { Mammography } \\
\text { BI-RADS }\end{array}$ & US BI-RADS & $\begin{array}{l}\text { Mammography/ } \\
\text { US BI-RADS }^{\mathrm{a}}\end{array}$ \\
\hline \multicolumn{4}{|c|}{ Non-malignant lesions $(n=75)$} \\
\hline BI-RADS 0 & 16 & 2 & \\
\hline BI-RADS 1 & 7 & 0 & \\
\hline BI-RADS 2 & 4 & 3 & \\
\hline BI-RADS 3 & 22 & 40 & 37 \\
\hline BI-RADS 4 & 21 & 22 & 29 \\
\hline BI-RADS 5 & 3 & 6 & 7 \\
\hline \multicolumn{4}{|c|}{ Malignant lesions $(n=101)$} \\
\hline BI-RADS 0 & 14 & 1 & \\
\hline BI-RADS 1 & 6 & 0 & \\
\hline BI-RADS 2 & 0 & 1 & \\
\hline BI-RADS 3 & 3 & 3 & 3 \\
\hline BI-RADS 4 & 19 & 20 & 15 \\
\hline BI-RADS 5 & 59 & 76 & 83 \\
\hline \multicolumn{4}{|c|}{ Negative breasts $(n=155)$} \\
\hline BI-RADS 0 & 47 & 31 & 27 \\
\hline BI-RADS 1 & 105 & 115 & 118 \\
\hline BI-RADS 2 & 2 & 5 & 6 \\
\hline BI-RADS 3 & 0 & 4 & 4 \\
\hline Total & 331 & 331 & 331 \\
\hline
\end{tabular}

${ }^{a}$ The higher BI-RADS value between mammography and US

images were viewed in cine-loop modality. The temporal curve of percent intensity change versus time was displayed on a grid when the user presses on the image pixels. The integrated software classifies the temporal curves pixel by pixel and these are displayed in different chromatic scales based on the curves' trend.

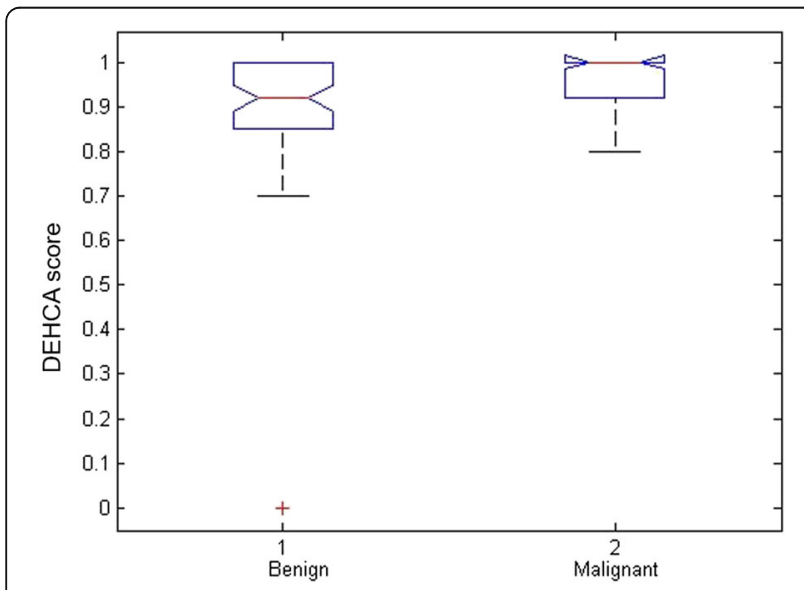

Fig. 9 Boxplot of DeHCA score to differentiate malignant versus non-malignant lesions

\section{DeHCA score}

A number of parameters were evaluated to obtain the DeHCA score [18]:

1. Focality description (FOC1). Presence of 'diffuse blue area' throughout the area surrounding the focal point; presence of 'different colored areas' (yellow, green, blue, etc.) and simultaneous presence of focal points; absence of purple colored areas; no focus (Fig. 1)

2. Blue nipple (BN). Moving to the frame where the focal area shows the darkest color and positioning the lesion marker and simultaneously analyzing the curve; by moving the cursor through the subsequent pixels and identifying the curve with the most negative attenuation value; checking whether the lesion marker overlaps with the nipple marker and removing the color if there are always dark areas at the two markers (vases, typical nipples, etc.) (Fig. 2)

3. Stability/mobility of the focal area related to the epicenter (EPI). Assesses whether, in respect to the marked lesion marker, the focal epicenter moves when the black area is formed or before its disappearance

4. Sharpness of the focal area (FOC2). Characteristics of the focal area with the maximum attenuation, using three degrees, as follows: (1) sharp focal area similar to a well-defined atoll sharp and compact dark spot inside the focal area; violet contour closely following the edges of the black spot, almost drawn; (2) intermediate focal area similar to an atoll, with less sharp borders than the previous case, compact black spot with pixel effect, violet spot not closely surrounding the edges of the black spot; (3) noncompact focal area, with the black spot loosely defined, black pixels spreading within the violet area, and the black/violet area expanding on a large portion of the overall area (Fig. 3)

5. Focal area size (AR). Ratio between lesion area and ellipse area calculated by the formula $\mathrm{AR}=(\mathrm{a} / 2) \times(\mathrm{b} / 2)$ $\times \pi$ (Fig. 4)

6. Maximum attenuation. Referring to the frame with maximum attenuation and visualizing the attenuation value positioning exactly on the lesion marker

7. Time of appearance (TEMP). Observing the previously positioned lesion marker, detecting the frame where the focal area starts forming near the marker in terms of a well-defined attenuation area; the color might start appearing light blue and become darker (Fig. 5)

8. Lesion curve typology. Classifying the lesion marker curve as one of the following: waving (integral value equal to null); rapidly descending (rapidly moves away); slowly descending; rapidly descending with bounce (where the final value varies more than $20 \%$ 
Table 5 Spearman's correlation coefficients

\begin{tabular}{|c|c|c|c|c|c|c|c|c|}
\hline & & DeHCA score & Mammography BI-RADS & US BI-RADS & ER & $P R$ & Ki67 & Herceptest \\
\hline \multirow[t]{2}{*}{ DeHCAscore } & Correlation coefficient & 1.0 & $0.221^{* *}$ & $0.152^{* *}$ & 0.072 & -0.093 & 0.094 & 0.026 \\
\hline & $p$ value & & 0.000 & 0.006 & 0.565 & 0.502 & 0.445 & 0.893 \\
\hline \multirow[t]{2}{*}{ Mammography BI-RADS } & Correlation coefficient & $0.229^{* *}$ & 1.0 & $0.629^{* *}$ & 0.046 & 0.105 & 0.191 & -0.005 \\
\hline & $p$ value & 0.000 & & 0.000 & 0.755 & 0.412 & 0.091 & 0.971 \\
\hline \multirow[t]{2}{*}{ US BI-RADS } & Correlation coefficient & $0.159^{* *}$ & $0.629^{* *}$ & 1.0 & -0.169 & -0.021 & -0.149 & 0.075 \\
\hline & $p$ value & 0.006 & 0.0 & & 0.256 & 0.943 & 0.228 & 0.532 \\
\hline \multirow[t]{2}{*}{ ER } & Correlation coefficient & 0.071 & 0.043 & -0.162 & 1.0 & $0.431^{* *}$ & 0.152 & 0.051 \\
\hline & $p$ value & 0.565 & 0.755 & 0.267 & & 0.001 & 0.244 & 0.681 \\
\hline \multirow[t]{2}{*}{$P R$} & Correlation coefficient & -0.091 & 0.105 & -0.024 & $0.411^{* *}$ & 1.0 & -0.132 & $-0.291^{*}$ \\
\hline & $p$ value & 0.512 & 0.411 & 0.862 & 0.001 & & 0.433 & 0.020 \\
\hline \multirow[t]{2}{*}{ Ki67 } & Correlation coefficient & 0.092 & 0.191 & -0.143 & 0.153 & -0.123 & 1.0 & $0.274^{*}$ \\
\hline & $p$ value & 0.442 & 0.093 & 0.228 & 0.243 & 0.432 & & 0.016 \\
\hline \multirow[t]{2}{*}{ Herceptest } & Correlation coefficient & 0.022 & -0.005 & 0.079 & 0.055 & $-0.291^{*}$ & $0.271^{*}$ & 1.0 \\
\hline & $p$ value & 0.891 & 1.0 & 0.534 & 0.682 & 0.022 & 0.016 & \\
\hline
\end{tabular}

US ultrasound, $E R$ estrogen receptor, $P R$ progesterone receptor

*Significant correlation, $p<0.05$

${ }^{* *}$ Significant correlation, $p<0.01$

from the value of maximum attenuation); slowly descending with final bounce (Fig. 6)

9. Presence of similar/dissimilar areas (SIM). Comparison between the lesion curve and curves in other regions of the breast to evaluate the presence of similar behaviors inside the breast (Fig. 7)

10. Bilaterality (BIL). Simultaneous presence of the following conditions: (1) the same maximum symmetry for both breast; (2) the same maximum attenuation (differentiation less 30\%); (3) the same curve type (Fig. 8)

The DeHCA score was calculated using the previous parameters with the formula reported in Table 1 $($ DeHCA score $=\mathrm{FOC} 1 \times \mathrm{BN} \times \mathrm{EPI} \times \mathrm{FOC} 2 \times \mathrm{AR} \times$ BIL $\times$ SIM $\times$ TEMP) in order to evaluate the probability of the presence of a breast cancer lesion. DeHCA score reporting time was 12 min (median value).

\section{Reference standard and pathological methods}

Each suspicious finding at mammography and/or US underwent pathology analysis. The reference standard was pathology from a surgical specimen for malignant lesions, pathology from a surgical specimen or core needle biopsy for benign lesions, and negative follow-up for contralateral negative breasts. Each patient work-up was decided following the joint decision of a multidisciplinary team, including at least one radiologist and one surgeon. Tumor and nodal stage were classified according to the system implemented by the American Joint Committee on Cancer staging [19]. The intensity, extent, and subcellular distribution of estrogen receptor (ER), progesterone receptor (PR), human epidermal growth factor receptor 2 (HER2), and Ki67 were evaluated as previously described [20]. The cutoff used to distinguish positive from negative cases was $\geq 1 \%$ for $E R / P R$ ratio. Scores of 0 or $1+$ were considered negative for HER2 expression, $2+$ and $3+$ scores were positive. In case of HER2 score equal to 2+, fluorescence in situ hybridization (FISH) amplification was performed and HER2 expression was considered as positive HER2 amplification when the FISH ratio was higher than 2.2 or the HER2 gene copy greater than 6.0; as equivocal HER2 amplification at a FISH ratio of $1.8-2.2$ or HER2 gene

Table 6 Diagnostic performance for discriminating breast diseases versus negative cases for mammography BI-RADS score, US BI-RADS score, mammography/US BI-RADS score, and DeHCA score

\begin{tabular}{|c|c|c|c|c|c|c|}
\hline & Area under the curve & Sensitivity & Specificity & Positive predictive value & Negative predictive value & $\overline{\text { Accuracy }}$ \\
\hline Mammography BI-RADS & 0.81 & 0.72 & 0.97 & 0.96 & 0.77 & 0.84 \\
\hline US BI-RADS & 0.96 & 0.94 & 0.96 & 0.96 & 0.94 & 0.95 \\
\hline Mammography/US BI-RADS & 0.98 & 0.96 & 0.95 & 0.95 & 0.96 & 0.96 \\
\hline DeHCA score (optimal cutoff $=0.85)$ & 0.55 & 0.62 & 0.47 & 0.54 & 0.56 & 0.55 \\
\hline DeHCA score $($ cutoff $=0.80)$ & 0.55 & 0.81 & 0.28 & 0.59 & 0.60 & 0.54 \\
\hline
\end{tabular}


Table 7 Diagnostic performance for detecting malignant lesions for mammography BI-RADS score, US BI-RADS score, mammography/US BI-RADS score, and DeHCA score

\begin{tabular}{|c|c|c|c|c|c|c|}
\hline & Area under the curve & Sensitivity & Specificity & Positive predictive value & Negative predictive value & Accuracy \\
\hline Mammography BI-RADS & 0.83 & 0.77 & 0.89 & 0.74 & 0.90 & 0.85 \\
\hline US BI-RADS & 0.96 & 0.96 & 0.87 & 0.76 & 0.98 & 0.90 \\
\hline Mammography/US BI-RADS & 0.97 & 0.98 & 0.84 & 0.72 & 0.99 & 0.88 \\
\hline DeHCA score (optimal cutoff $=0.85$ ) & 0.65 & 0.78 & 0.52 & 0.40 & 0.85 & 0.60 \\
\hline DeHCA score $($ cutoff $=0.80)$ & 0.65 & 0.93 & 0.32 & 0.37 & 0.91 & 0.50 \\
\hline
\end{tabular}

copy of 4.0-6.0; and as negative HER2 amplification when the FISH ratio was lower than 1.8 or HER2 gene copy less than 4.0. The percentage of positive cells per case for proliferative index Ki67 was scored according to two different groups - group $1,<15 \%$ (low proliferative activity, negative cases); group $2, \geq 15 \%$ (high proliferative activity, positive cases). Ductal carcinoma in situ and invasive cancer tumors were counted as malignant lesions. All other results, including lobular carcinoma in situ, fibroadenoma, ductal hyperplasia, dysplasia, cysts, and phyllodes tumors, were considered nonmalignant lesions.

\section{Statistical analysis}

The DeHCA score performance was evaluated by using both the optimal cutoff value (0.85) and also the 0.80 value (to maximise sensitivity). Continues variables were reported as median and 75th and 25th percentile (interquartile range, IQR). Receiver operating characteristic (ROC) analysis was used to define the optimal cutoff maximizing the Youden index. Sensitivity, specificity, $\mathrm{PPV}$, negative predictive value (NPV), and accuracy were calculated for mammography, US, combined mammography and US, and DeHCA score. For inter-group comparisons, we used the Mann Whitney $U$ test for continuous variables and $\chi^{2}$ test for categorical variables. Spearman's correlation coefficient was also calculated to compare DeHCA score and BI-RADS score with pathological findings. Additionally, we assessed the diagnostic accuracy for a linear classifier that combines DeHCA score with mammography $([a \times$ DeHCA score $]+[b \times$ mammography BI-RADS category] $>$ constant) and for a linear classifier that combines DeHCA score with US mammography $([a \times$ DeHCA score $]+[b \times$ US BI-RADS category $]$
$>$ constant). The linear classifier of the Statistics and Machine Learning Toolbox of Matlab R2007 individuates the coefficient of linear combination and the constant; when the weighted sum exceeds the constant, the classifier returns a test positive case. McNemar test was used to compare the diagnostic performance in terms of sensitivity and specificity. A $p$ value of less than 0.05 was considered significant. Calculations were performed using the Statistics and Machine Learning Toolbox of Matlab R2007a (MathWorks, Natick, USA).

\section{Results}

Of 334 enrolled patients, 168 patients (50\%) were excluded for technical problems, 94 patients $(28 \%)$ for technical failure at optical image processing, $73(22 \%)$ due to having too small or large breasts, and 1 for having breasts that were too dense. Therefore, 166 (50\%) patients entered the analysis, with a median age of 52 years (IQR 64-44 years). A total of 176 lesions and 331 breasts were investigated (one breast for one patient was eliminated for absence of cytological characterization in the work-up).

Overall, at pathology, 75 lesions were benign (median size $19 \mathrm{~mm}, \mathrm{IQR} 24-11 \mathrm{~mm}$ ) and 101 were malignant (median size $20 \mathrm{~mm}$, IQR $26-16 \mathrm{~mm}$ ), and a total of 155 breasts had negative follow-up at conventional imaging (Table 2). The distribution of pathology prognostic factors is provided in Table 3. For seven patients with Herceptest status equal to 2+, FISH analysis was performed, returning three positive cases and four negative cases. There were significant differences between groups in terms of age and positive versus negative ER/PR expression, while there were no significant differences between groups in terms of grading, Herceptest status, and for

Table 8 Diagnostic performance of DeHCA score plus mammography linear combination and of DeHCA score plus US linear combination

\begin{tabular}{|c|c|c|c|c|c|c|}
\hline & Area under the curve & Sensitivity & Specificity & Positive predictive value & Negative predictive value & Accuracy \\
\hline \multicolumn{7}{|c|}{ To discriminate breast disease versus negative cases } \\
\hline DeHCA plus mammography & 0.78 & 0.72 & 0.96 & 0.94 & 0.78 & 0.84 \\
\hline DeHCA plus US & 0.93 & 0.94 & 0.96 & 0.96 & 0.94 & 0.95 \\
\hline \multicolumn{7}{|l|}{ To detect malignant lesions } \\
\hline DeHCA plus mammography & 0.73 & 0.93 & 0.82 & 0.89 & 0.87 & 0.73 \\
\hline DeHCA plus US & 0.96 & 0.92 & 0.81 & 0.98 & 0.93 & 0.96 \\
\hline
\end{tabular}


positive and negative Ki67. BI-RADS score distribution was reported in Table 4.

The DeHCA score of malignant lesions (median 0.95, IQR 1.0-0.87) was significantly higher than that of benign lesions (median 0.8, IQR 0.95-0.7) ( $p<0.001$, Mann Whitney $U$ test, see also boxplot of DeHCA score in Fig. 9). Table 5 reports the Spearman's correlation coefficients to compare the DeHCA and BI-RADS scores with pathological findings. No correlations were observed.

Table 6 reports the diagnostic performance of mammography, US, mammography/US, and DeHCA score in the detection of breast abnormalities, including benign and malignant cases versus negative cases (no abnormalities). Table 7 reports the detection of malignant lesions.

Figures 10 and 11 show the ROC curves for both classification analyses. DeHCA optical image processing showed a lower accuracy compared to mammography, US, and their combination using either the optimal cutoff value $(0.85)$ or the cutoff of 0.8 . Table 8 reports the diagnostic performance of the linear classifier that combines DeHCA score with mammography and of the linear classifier that combines DeHCA score with US. Figure 12 shows ROC curves for both linear classifiers to (1) discriminate breast diseases from negative cases and (2) detect malignant lesions.

The linear combination of DeHCA score and mammography and the linear combination of DeHCA score and US showed a significantly higher accuracy than DeHCA score alone $(p<0.001$, McNemar test) both to discriminate breast diseases from negative cases and to distinguish malignant lesions; the increase in accuracy was not significant compared to mammography alone or US alone (DeHCA score plus mammography versus mammography alone, $p=0.421$; DeHCA score plus US

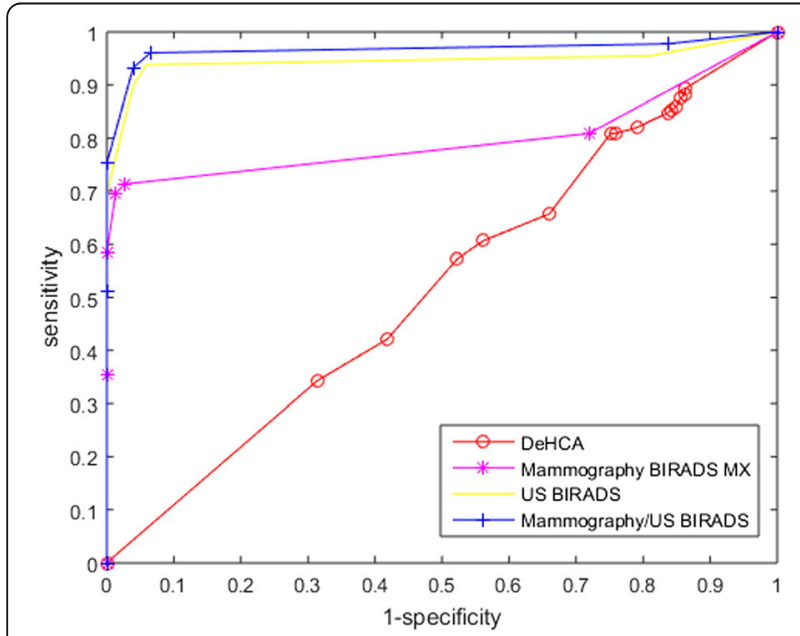

Fig. 10 ROC curve in the discrimination of breast diseases including non-malignant and malignant cases versus negative ones

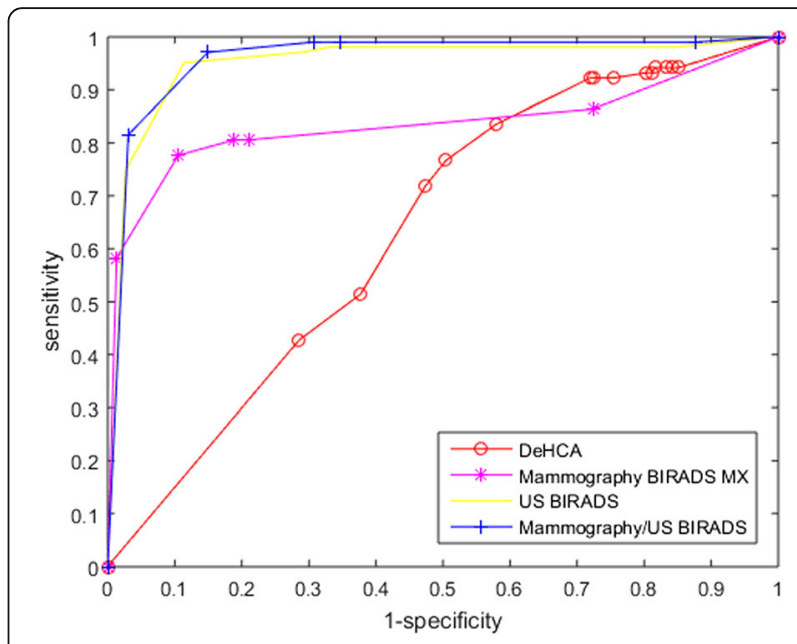

Fig. 11 ROC curve to discriminate malignant lesions

versus US alone, $p=0.302$; both McNemar test). Moreover, DeHCA score combined linearly to US in the detection of malignant lesions showed a higher accuracy $(93 \%)$, although not statistically significant, than mammography alone (85\%) and a higher sensitivity (96\%) than mammography alone (77\%) ( $p=0.09$, McNemar test).

A false negative at mammography was shown to be a true positive at DeHCA optical image processing (score 0.85) (Fig. 13). With US, a hypoechoic nodule was observed in the outer quadrant of the left breast with irregular borders of $12 \times 6 \mathrm{~mm}$ with calcifications inside (Fig. 14). Mammography showed a heterogeneous hyperdense lesion with irregular borders of approximately 30 $\mathrm{cm}$ in diameter. DeHCA optical image processing gave a true positive result (score 0.92).

\section{Discussion}

Dynamic optical breast imaging may identify breast tumors by detecting changes in local blood perfusion and oxygen saturation due to neoangiogenesis. The dynamic optical breast imaging has numerous potential advantages, including the use of non-ionizing, low-energy light radiation, continuous data acquisition for real-time monitoring, and low cost. To date, few papers investigating the use of the dynamic optical breast imaging for the early detection of breast cancer are available.

Athanasiou et al. [13] reported the results in a series of 72 patients with BI-RADS score 4-5 breast lesions. The diagnostic accuracy reached by the optical technology applied to BI-RADS score 4 or 5 breast lesions was $73 \%$ sensitivity and $38 \%$ specificity. False negatives were mostly related to small lesions $(<10 \mathrm{~mm})$, while false positives were mainly benign proliferative lesions. The interpretation of optical imaging was based on the analysis of the presence of early, focal, intense blue color in the area of interest, the pixel intensity of the blue 

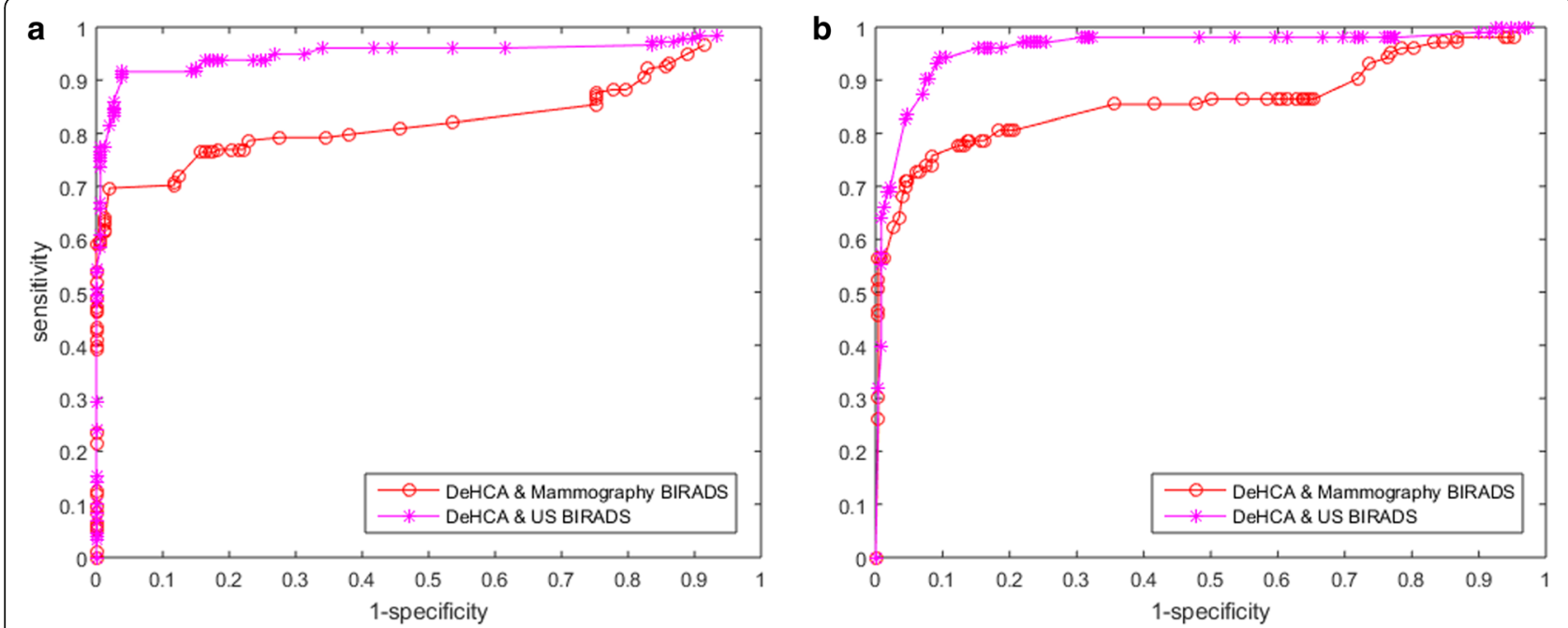

Fig. 12 ROC curves for both linear classifiers (DeHCA score and mammography and DeHCA score and US) to (a) discriminate breast diseases versus negative cases and (b) differentiate malignant from non-malignant breast lesions

areas, and the type of temporal signature of dynamic curves. A numeric level of suspicious score was calculated based on all these elements and a score more than 5 was considered a suspect. However, the criteria of imaging interpretation were not clearly defined and there was a high interobserver variability in the determination of the score.

Frattini et al. [21] performed a prospective analysis of 617 young women evaluating the diagnostic accuracy of the combined use of optical technology and US, and found a sensitivity of $97 \%$ and specificity of $87 \%$, a net increase in accuracy compared to US alone (sensitivity $74 \%$, specificity 70\%). Fournier et al. [14] evaluated the dynamic optical breast imaging system in 47 BI-RADS score 3-5 breast lesions. A significant difference in numbers of suspect pixels between 12 benign lesions and 35 malignant lesions was observed; the ROC curve showed an optimal cutoff for the number of pixels equal to 2050, which determined a sensitivity of $26 / 35$ (74\%) and a specificity of $11 /$ 12 (92\%). Of note, 6 out of 9 malignant lesions missed by dynamic optical breast imaging had been classified BIRADS score 5 by mammography. In view of that, the authors stated that mammography and optical imaging should be considered complementary, as they describe different physiological properties of tissues.

Considering that, to date, breast diagnosis relies on the combined use of complementary technologies, the aim of this study is to determine the diagnostic performance of an evolution of dynamic breast optical technology in patients with breast lesions using a score system and the combination of dynamic breast optical imaging and conventional imaging (mammography or US).

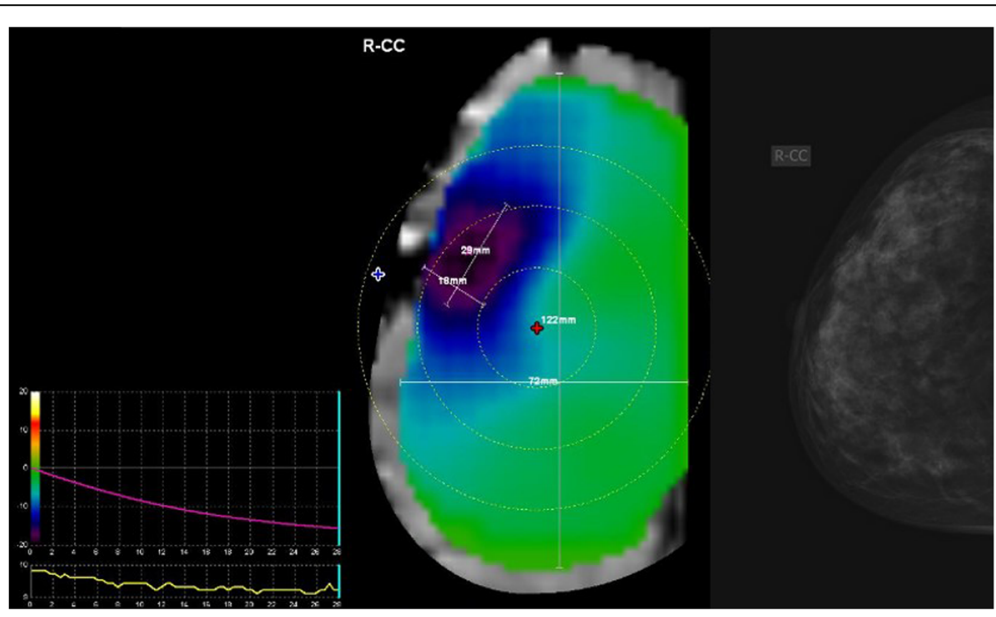

Fig. 13 Mammography appearance with prevalent, fibroglandular component most represented in the superior external quadrant, with consequent limited diagnostic definition. There is no obvious focal opacity. Instead, the DeHCA score was equal to 0.85 . Final diagnosis: invasive ductal carcinoma 


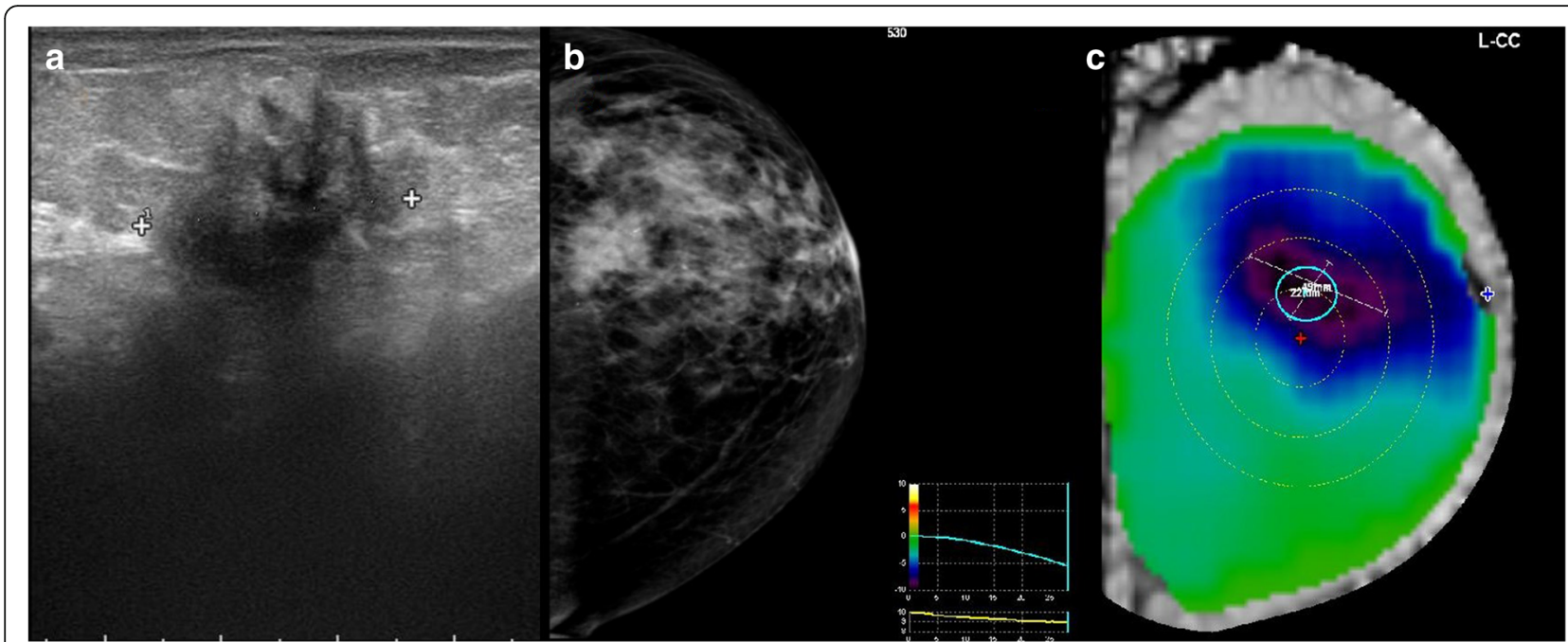

Fig. 14 Mammography (a) shows a heterogeneous finding with irregular edges of approximately $30 \mathrm{~cm}$ in diameter. DeHCA score (b) was equal to 0.92. At US (c), in the outer quadrant of the left breast, a hypoechoic nodule with irregular borders is visible, measuring $12 \times 6 \mathrm{~mm}$, with internal dot-like calcifications. Final diagnosis: invasive ductal carcinoma

We reported a statistical significant difference in DeHCA score median value for benign versus malignant breast lesions ( $p<0.001$, Mann Whitney $U$ test) but no correlations were observed between DeHCA and BIRADS scores as well as with the pathological findings. The measurement of the DeHCA biomarker made using Comfortscan images showed a lower accuracy than mammography, US, and their combination using the optimal cutoff (0.85) obtained by ROC analysis, with $78 \%$ sensitivity, 52\% specificity, 40\% PPV, and 85\% NPV; using a threshold of 0.8 , sensitivity reached $93 \%$ and NPV 91\%, but specificity fell to only $32 \%$ and PPV to only $37 \%$.

The linear combination of DeHCA score and mammography and the linear combination of DeHCA score and US showed a significantly higher accuracy than DeHCA score based on Comfortscan alone; the increase in accuracy was not significant compared to mammography alone or US alone. Moreover, DeHCA score combined linearly to US in the detection of malignant lesions showed a higher accuracy (93\%) than mammography alone (85\%) and a higher sensitivity (96\%) than mammography alone (77\%; $p=0.09$, McNemar test).

In this perspective, DeHCA optical image processing may play a role in breast assessment in combination with US, although the additional diagnostic contribution of DeHCA optical image processing to that of US alone deserves further investigation.

There are some limitations to our study that must be considered. The high exclusion percentage for technical problems $(50 \%)$ is as a limitation of the current optical imaging procedure investigated herein. However, technological improvements are being continually developed to reduce the number of eliminations. Particularly in the early phase of the study, we had several technical failures mainly due to the use of ComfortScan hardware, which is an evolving digital optical scanner. Breast imaging was acquired only in cranio-caudal projection, which may cause an underdetection of lesions localized in the axillary pilaster and/or mammary sulcus. Moreover, DeHCA optical image processing was not feasible in some women with very small, firm breasts that could not be properly illuminated as well as in women previously submitted to surgery and/or bioptic procedures due to the presence of residual edema and extravasation. The possible limiting role of factors like inflammatory breast conditions, skin breast tattoos, menstrual cycle phases, and vasculitis in the appropriate DeHCA optical image processing should be addressed in appropriate trials. The overall disease prevalence in our series, and the high number of malignant versus benign lesions among the positive cases, is not truly representative of the female population normally investigated in a screening breast imaging center. This was due to the second level nature of our cancer center and to the need to have a pathological standard reference available for the study purposes. In addition, not all cases had both mammography and US available. Finally, it must also be considered that we used film-screen mammography, which is no longer considered the state-of-the art technique, despite still being utilized in many screening programs.

In conclusion, in our experience, optical imaging provided a DeHCA score in malignant lesions that was significantly higher than that of benign lesions. However, using the optimal cutoff $(0.85)$ or a threshold of 0.8 for 
DeHCA score, accuracy remained significantly lower than mammography or US alone, not allowing for a stand-alone use of the current status of this technology in breast cancer diagnosis. The linear combination of DeHCA score and mammography and of DeHCA score and US significantly increased the diagnostic performance accuracy with respect to DeHCA score alone. Future technological refinements are needed to make this technology ready for clinical practice. In this possible future scenario, DeHCA technology in combination with US might be an interesting perspective.

\section{Abbreviations}

AR: focal area size; BIL: bilaterality; BN: blue nipple; CCD: charge coupled device; DeHCA: deoxyhemoglobin concentration alteration; EPI: stability/ mobility of the focal area related to the epicenter; ER: estrogen receptor; FISH: fluorescence in situ hybridization; FOC1: focality description; FOC2: sharpness of the focal area; HER2: human epidermal growth factor receptor 2; IQR: interquartile range; LED: light-emitting diode; NPV: negative predictive value; PPV: positive predictive value; PR: progesterone receptor; ROC: receiver operating characteristic; SIM: presence of similar/dissimilar areas; TEMP: time of appearance; US: ultrasound

\section{Acknowledgements}

The authors are grateful to Alessandra Trocino, librarian at the National Cancer Institute of Naples, Italy.

\section{Funding}

Not applicable.

\section{Availability of data and materials}

All data generated or analyzed during this study are included in this published article.

\section{Authors' contributions}

Each author has participated sufficiently in this submission and takes public responsibility for its content. All authors read and approved the final manuscript.

\section{Ethics approval and consent to participate}

This study was performed according to regulations issued by our local Institutional Review Board.

\section{Consent for publication}

All patients provided informed consent for the use of their data for research purposes.

\section{Competing interests}

The authors declare that they have no competing interests.

\section{Publisher's Note}

Springer Nature remains neutral with regard to jurisdictional claims in published maps and institutional affiliations.

\section{Author details}

'Radiology Unit, Dipartimento di Supporto ai Percorsi Oncologici Area Diagnostica, Istituto Nazionale Tumori - IRCCS - Fondazione G. Pascale, Via Mariano Semmola, Naples, Italy. ${ }^{2}$ Senology Surgery Unit, Dipartimento Corp-S Assistenziale e di Ricerca dei Percorsi Oncologici del Distretto Toracico, Istituto Nazionale Tumori - IRCCS - Fondazione G. Pascale, Via Mariano Semmola, Naples, Italy. ${ }^{3}$ Pathology Unit, Dipartimento di Supporto ai Percorsi Oncologici Area Diagnostica, Istituto Nazionale Tumori - IRCCS - Fondazione G. Pascale, Via Mariano Semmola, Naples, Italy.
Received: 9 November 2017 Accepted: 31 January 2018

Published online: 23 April 2018

\section{References}

1. Breast Cancer Estimated Incidence, Mortality and Prevalence Worldwide in 2012 (2012). http://ww5.komen.org/BreastCancer/Statistics.html. Accessed 21 Mar 2017.

2. Caplan L (2014) Delay in breast cancer: implications for stage at diagnosis and survival. Front Public Health 2:87

3. Weedon-Fekjær H, Romundstad PR, Vatten $\sqcup$ (2011) Modern mammography screening and breast cancer mortality: population study. BMJ 348:93701

4. Heywang-Köbrunner SH, Hacker A, Sedlacek S (2011) Advantages and disadvantages of mammography screening. Breast Care 6:199-120

5. Gøtzsche PC, Jørgensen KJ (2013) Screening for breast cancer with mammography. Cochrane Database Syst Rev 6:CD001877

6. Kerlikowske K, Carney PA, Geller B et al (2000) Performance of screening mammography among women with and without a first-degree relative with breast cancer. Ann Intern Med 133:855-863

7. Corsetti V, Houssami N, Ghirardi M et al (2011) Evidence of the effect of adjunct ultrasound screening in women with mammography-negative dense breasts: interval breast cancers at 1 year follow-up. Eur J Cancer 47:1021-1026

8. Thompson DJ, Leach MO, Kwan-Lim G et al (2009) UK study of MRI screening for breast cancer in women at high risk (MARIBS). Assessing the usefulness of a novel MRI-based breast density estimation algorithm in a cohort of women at high genetic risk of breast cancer: the UK MARIBS study. Breast Cancer Res 1:R80

9. Podo F, Sardanelli F, Canese R et al (2002) The Italian multi-centre project on evaluation of MRI and other imaging modalities in early detection of breast cancer in subjects at high genetic risk. J Exp Clin Cancer Res 21:115-124

10. Panizza P, Viganò S, Bonelli L et al (2012) Screening women at intermediate risk: harm or charm? Eur J Radiol 81:S116-S117

11. Cutler M (1929) Transillumination as an aid in the diagnosis of breast lesions. Surgery Gynecol Obstet 48:721-729

12. Arora N, Martins D, Ruggerio D et al (2008) Effectiveness of a noninvasive digital infrared thermal imaging system in the detection of breast cancer. Am J Surg 196:523-526

13. Athanasiou A, Vanel D, Balleyguier C et al (2005) Dynamic optical breast imaging: a new technique to visualise breast vessels: comparison with breast MRI and preliminary results. Eur J Radiol 54:72-79

14. Fournier $L S$, Vanel $D$, Athanasiou A et al (2009) Dynamic optical breast imaging: a novel technique to detect and characterize tumor vessels. Eur J Radiol 69:43-49

15. Cheng L, Li J, Liu M et al (2011) Comparison of dynamic optical breast imaging (DOBI) and mammography in sensitivity, specificity and safety of breast cancer diagnosis: a prospective analysis of 62 patients in two centers. Beijing Da Xue Xue Bao 43:467-471

16. D'Aiuto M, Frasci G, Barretta ML et al (2012) The dynamic optical breast imaging in the preoperative workflow of women with suspicious or malignant breast lesions: development of a new comprehensive score. ISRN Oncol 2012:631917

17. American College of Radiology (1995) Breast Imaging Reporting and Data System (BI-RADS), 2nd edn. American College of Radiology, Reston

18. Zhang JG (2013) ComfortScan ${ }^{T M}$ system clinical effectiveness evaluation report. http://dobiglobal.com/pdfs/DOBI-ANewTechniqueToDetectBreastCancerlnEarly Stage_ENG.pdf. Accessed 2 Oct 2017

19. American Joint Committee Cancer (2002) AJCC Cancer staging manual, 6th edn. Springer, Chicago

20. Crispo A, Barba M, D'Aiuto $G$ et al (2013) Molecular profiles of screen detected vs. symptomatic breast cancer and their impact on survival: results from a clinical series. BMC Cancer 13:15

21. Frattini V Ghisoni L, Teodoro A et al (2011) Clinical approach with optical imaging instrument. Perspective analysis on 617 young females. It $J$ Gynæcol Obstet 23:101-106 